\title{
BMJ Open Multicentre randomised phase II study of the perioperative administration of flurbiprofen axetil in patients with non- small cell lung cancer: study protocol of the FLAX Study
}

Kentaro Sakamaki, ${ }^{1}$ Katsuya Watanabe (D) ${ }^{2}$ Tetsukan Woo, ${ }^{3}$ Munetaka Masuda ${ }^{4}$

To cite: Sakamaki K, Watanabe K, Woo T, et al. Multicentre randomised phase II study of the perioperative administration of flurbiprofen axetil in patients with non-small cell lung cancer: study protocol of the FLAX Study. BMJ Open 2020;10:e040969. doi:10.1136/ bmjopen-2020-040969

- Prepublication history for this paper is available online. To view these files, please visit the journal online (http://dx.doi. org/10.1136/bmjopen-2020040969).

Received 31 May 2020 Revised 06 November 2020 Accepted 09 November 2020

\section{Check for updates}

(c) Author(s) (or their employer(s)) 2020. Re-use permitted under CC BY-NC. No commercial re-use. See rights and permissions. Published by BMJ.

${ }^{1}$ Center for Data Science, Yokohama City University, Yokohama, Japan

${ }^{2}$ General Thoracic Surgery, National Hospital Organisation Yokohama Medical Center, Yokohama, Japan

${ }^{3}$ Respiratory Center, Yokohama City University Medical Center, Yokohama, Japan

${ }^{4}$ Surgery, Yokohama City University, Yokohama, Japan

Correspondence to Dr Katsuya Watanabe; watanabe.katsuya.gt@mail. hosp.go.jp

\section{ABSTRACT}

Introduction In patients with non-small cell lung cancer, surgical treatment with postoperative adjuvant chemotherapy is performed. However, the improvement of overall survival achieved by postoperative adjuvant chemotherapy may be insufficient in consideration of the deterioration of quality of life (QOL). Considering the relationships among surgical treatments, inflammation and carcinogenesis, non-steroidal anti-inflammatory drugs (NSAIDs) are a candidate postoperative treatment for preventing recurrence and maintaining QOL. In this study, we investigate the effects of the perioperative administration of flurbiprofen axetil on postoperative recurrence in patients with non-small cell lung cancer. Methods and analysis This study is a multicentre, parallel group, open label, randomised controlled trial. Patients clinically suspected of non-small cell lung cancer are randomly assigned to the flurbiprofen axetil group or the no-NSAIDs group. A total of 420 patients (210 per group) will be registered. The primary analysis will evaluate the treatment effect of flurbiprofen axetil on postoperative recurrence.

Ethics and dissemination The study protocol was approved by the Clinical Research Review Board of Saitama Medical University in September 2019 (№. 192002) and will be approved by each institutional review board of all participating institutions before patient enrolment. This study complies with the latest version of the Declaration of Helsinki, Clinical Trial Act and related notifications. Results will be published in a peer-reviewed journal.

Trial registration number Japan Registry of Clinical Trials (jRCTs031190167; Pre-results) (https://jrct.niph.go.jp/).

\section{BACKGROUND}

Lung cancer is the leading cause of cancer death worldwide. ${ }^{12}$ In Japan, non-small cell lung cancer (NSCLC) accounts for $75 \%-85 \%$ of all lung cancers. ${ }^{3}$ Although there have been no randomised controlled trials to compare surgery with radiation therapy or chemotherapy, observational studies have suggested that surgery is the most curative treatment
Strengths and limitations of this study

- This is the first study to evaluate the effect of the perioperative administration of non-steroidal antiinflammatory drugs (NSAIDs) on postoperative recurrence in non-small cell lung cancer.

- Flurbiprofen axetil is a safe and inexpensive NSAID used perioperatively as a part of multimodal analgesia.

- This study is a multicentre, parallel group, open label, randomised controlled trial.

- The lack of blinding of patients and specialists during intervention may be a limitation.

for lung cancer. ${ }^{45}$ However, surgery alone may not be a sufficient treatment because an observational study of 11663 surgically treated patients with NSCLC showed that the 5-year overall survival (OS) rate in all patients was $69.6 \%$, while the rates in patients with clinical stage IA, IB, IIA and IIB were $82.0 \%, 66.1 \%, 54.5 \%$ and $46.1 \%$, respectively. ${ }^{2}$ Even in completely resected cases, approximately $50 \%$ of patients showed local metastasis and/or distant metastases due to micrometastasis. ${ }^{67}$ Thus, postoperative adjuvant chemotherapy has been performed. In Japan, postoperative adjuvant chemotherapy regimens include tegafur-uracil combination therapy for pathological stage IA3 and IB, ${ }^{89}$ and cisplatin combination chemotherapy for pathological stage II-IIIA. ${ }^{10-12}$ Although postoperative adjuvant chemotherapy can only improve the 5 -year OS rate by $4 \%-5 \%,{ }^{8-12}$ the use of cytotoxic anticancer drugs during the postoperative phase may be associated with deterioration of the patient's quality of life. Therefore, it is important to establish a new perioperative treatment to prevent recurrence and maintain quality of life. 
Surgical treatment can cause a sharp inflammatory response due to local tissue damage. The relationship between carcinogenesis and inflammation is empirically known. ${ }^{13}$ For example, a relationship between chronic inflammation due to infection and carcinogenesis can be seen with the following conditions: hepatocellular carcinoma and hepatitis $\mathrm{C}$ virus, gastric cancer and Helicobacter pylori, and cervical cancer and human papilloma virus. ${ }^{14}$ Although the duration, cause and pathology of inflammation differ, these findings suggest that a common molecular mechanism may exist during the carcinogenesis process. ${ }^{15}$ For example, various inflammatory cytokines, chemical mediators such as prostaglandins, and growth factors, such as vascular endothelial growth factor (VEGF) are involved when promoting growth, proliferation and metastasis of cancer cells. ${ }^{16}$ Surgical invasion causes the production and secretion of inflammatory cytokines, prostaglandins and VEGF, which are responsible for the regulation of various physiological functions during the wound healing process. Thus, surgical inflammation may contribute to early postoperative recurrence. ${ }^{17}$ In fact, a high neutrophil to lymphocyte ratio (NLR) was reported to be associated with a poor prognosis in 255 patients with lung cancer. ${ }^{18}$ In tumor-bearing mice, Krall et $a l^{19}$ demonstrated that surgical invasion accelerates cellular proliferation and distant metastasis in patients with micrometastasis and that meloxicam suppresses these processes.

Non-steroidal anti-inflammatory drugs (NSAIDs) have various pharmacological actions, including antiinflammatory and analgesic actions. While the role of NSAIDs in cancer prevention remains unclear due to inconsistent and contradicting findings, ${ }^{20}$ several observational studies have shown that carcinogenesis seems to be suppressed in regular users of NSAIDs, ${ }^{21}$ and this association was reported in various cancer types, ${ }^{22}$ including colon cancer with high cyclooxygenase-2 (COX-2) expression levels ${ }^{23}$ and lung cancer. ${ }^{24}$ Flurbiprofen axetil (Ropion) is the only intravenous non-steroidal analgesic in Japan and a prodrug of flurbiprofen that strongly inhibits prostaglandin biosynthesis. Although COX-2 selectivity of flurbiprofen axetil is not high as ketorolac which is widely used in Europe and the USA, it is an inexpensive analgesic and is widely used as an adjunct for general anaesthesia. A meta-analysis ${ }^{25}$ showed that flurbiprofen axetil is useful and safe for postoperative analgesia, and some studies have reported that it suppresses postoperative elevations of inflammatory cytokines and growth factors in blood ${ }^{26} 27$ and that intraoperative use of it with steroids might suppress postoperative recurrence of lung cancer. ${ }^{28}$

It has been pointed out that anaesthesia, analgesia and perioperative management may affect the postoperative recurrence and long-term prognosis of patients with cancer. ${ }^{29}{ }^{30}$ Forget et al reported that anaesthetic management using NSAIDs was associated with the long-term prognosis of patients with breast cancer who received curative surgery. In a retrospective study of 319 patients with mastectomy with axillary dissection, the recurrence rate in the ketorolac (NSAIDs) group was decreased in comparison to the no ketorolac group $(\mathrm{HR}=0.37$, $\mathrm{p}=0.019) .{ }^{31}$ In consideration of this finding, a randomised controlled trial is currently underway in Europe to determine whether the intraoperative administration of ketorolac contributes to postoperative recurrence in patients with breast cancer. ${ }^{32}$ However, there have been no attempts to verify whether the perioperative administration of NSAIDs contributes to postoperative recurrence in patients with lung cancer. In this multicentre randomised phase II study, we investigate the effect of the perioperative administration of flurbiprofen axetil on postoperative recurrence in NSCLC.

\section{Study objectives}

The objective of the present study is to evaluate efficacy and safety of the perioperative administration of flurbiprofen axetil for preventing recurrence of NSCLC.

\section{Trial design}

This study is a multicentre, parallel group, open label, randomised controlled trial. The registration period is between December 2019 and September 2021, the planned follow-up period is at least 5 years.

\section{METHODS AND ANALYSIS \\ Patient selection (eligibility criteria)}

Patients with clinically suspected NSCLC are eligible for this study if they meet the following criteria. The inclusion criteria are as follows: (1) age $\geq 20$ years; (2) Eastern Cooperative Oncology Group performance status, 0-2; (3) clinical stage IA-IIIA; (4) radiologically diagnosed invasive lung tumour with a solid component of $\geq 1.0 \mathrm{~cm}$ visualised by thin-section CT; (5) complete resection, including mediastinal lymph node dissection or sampling is planned; (6) neutrophil count $\geq 1500 \mu \mathrm{L}$; (7) haemoglobin $\geq 80 \mathrm{~g} / \mathrm{L}$; (8) platelet count $\geq 100 \times 10^{9} / \mathrm{L}$; (9) aspartate transaminase $\leq 80 \mathrm{IU} / \mathrm{L}$ and alanine transaminase $80 \mathrm{IU} / \mathrm{L}$; (10) serum creatinine $\leq 1.5 \mathrm{mg} / \mathrm{dL}$; (11) $\mathrm{SpO}_{2}$ $\geq 92 \%$ (room air). The tumours are staged according to the eighth edition of the Union for International Cancer Control TNM Classification of Malignant Tumors. ${ }^{33}$

The exclusion criteria are as follows: (1) active double cancer; (2) history of surgery, chemotherapy or radiation within 5 years; (3) serious complications; (4) pregnant or lactating; (5) regular NSAID users who have difficulty stopping NSAID use between 1 week before surgery and discharge; (6) NSAID allergy; (7) systemic administration of steroids or immunosuppressants; (8) administration of enoxacin hydrate, lomefloxacin, norfloxacin or prulifloxacin.

\section{Participating institutions}

Planned participating institutions are as follows: National Hospital Organization Yokohama Medical Center, Yokohama City University Hospital, Yokohama City University 
Medical Center, Kanagawa Cardiovascular and Respiratory Center, Yokosuka Kyosai Hospital, Yokohama Minami Kyosai Hospital, Hiratsuka Kyosai Hospital, Yokohama Rosai Hospital, Kanto Rosai Hospital, Yokosuka General Hospital Uwamachi, Saiseikai Yokohamashi Nanbu Hospital (Yokohama Consortium of Thoracic Surgeons); and Hamamatsu University Hospital, Teikyo University Mizonokuchi Hospital, Saitama Cardiovascular and Respiratory Center (The International Lung-ClinicalStudy Organization).

\section{Randomisation}

Eligible patients are randomly assigned to the flurbiprofen axetil group or the no-NSAIDs group by a minimisation method using (1) institution, (2) clinical stage (IA1, 2/ IA3, IB/IIA or more), and (3) sex because postoperative adjuvant chemotherapy varies according to stage, and the prognosis varies according to sex. ${ }^{3435}$ The randomisation ratio is $1: 1$. The results of the assignment will not be made known to the study subjects.

\section{Blinding}

As the study drugs were administrated not only intravenously but also orally throughout the perioperative period, the blinding of the patients, surgeons and anesthesiologists was considered difficult due to the nature of the interventions, so we chose to conduct an open-label trial. However, in order to minimise the risk of bias in the outcome assessments, cancer recurrence will be diagnosed with at least one independent radio-oncologist or pulmonologist. The statistician analysing the results will also be blinded to group allocation.

\section{Treatments}

\section{Flurbiprofen axetil group}

Flurbiprofen axetil (50 mg) is slowly injected intravenously between the induction of general anaesthesia and surgical incision. The use of NSAIDs as oral analgesics is recommended postoperatively. Other analgesic methods, such as epidural anaesthesia, intravenous patientcontrolled analgesia (IVPCA) and intercostal nerve block, are selected based on the clinical practice of each anaesthesiologist. The repeated administration of flurbiprofen axetil injection is also allowed at least 6 hours apart as part of a multimodal approach to achieve postoperative pain relief until effective pain relief can be administered.

\section{No-NSAIDs group}

Analgesic methods other than the systemic administration of NSAIDs (suppositories, intravenous injections) should be selected based on clinical practice. For postoperative multimodal analgesia, acetaminophen alone or tramadol hydrochloride/acetaminophen combination products are used together with epidural anaesthesia, IVPCA or intercostal nerve block. In principle, NSAIDs are not used during hospitalisation; however, the systemic administration of acetaminophen preparations (suppositories, intravenous injections) and the use of NSAIDs patches to provide sufficient analgesia are acceptable.

\section{Assessment measures}

Assessments to be performed at enrolment, at surgery and for 5 years after surgery based on the Japanese guidelines $^{36}$ are shown in table 1 . Examinations and imaging tests are performed every 3 months until 3 years after surgery, and every 6 months thereafter. Extraordinary assessments based on patient reports are permitted. It

Table 1 Overview of assessments

\begin{tabular}{|c|c|c|c|c|c|c|}
\hline Time point & $\begin{array}{l}2 \text { months to } \\
\text { day of surgery }\end{array}$ & $\begin{array}{l}\text { Day of } \\
\text { surgery }\end{array}$ & $\begin{array}{l}\text { Postop } 1 \\
\text { month }\end{array}$ & $\begin{array}{l}\text { Every } 3 \text { months } \\
\text { for } 3 \text { years }\end{array}$ & $\begin{array}{l}\text { Every } 6 \text { months } \\
\text { for } 5 \text { years }\end{array}$ & $\begin{array}{l}\text { Every } 12 \text { months } \\
\text { for } 5 \text { years }\end{array}$ \\
\hline Eligibility screen & $x$ & & & & & \\
\hline Informed consent & $x$ & & & & & \\
\hline Allocation & & $\mathrm{x}$ & & & & \\
\hline Medical history & $x$ & & & & & \\
\hline Physical examination & $x$ & & & & & $x$ \\
\hline Haematology & $\mathrm{x}$ & & & & & $\mathrm{x}$ \\
\hline Blood chemistry & $x$ & & & & & $x$ \\
\hline ECG & $x$ & & & & & \\
\hline Pulmonary function test & $x$ & & & & & \\
\hline Chest X-ray & & & & $x$ & & \\
\hline Chest and abdominal CT & & & & & $x$ & \\
\hline Perioperative analgesia & & & $\mathrm{x}$ & & & \\
\hline Postoperative complications & & & $x$ & & & \\
\hline Cancer recurrence ${ }^{*}$ & & & & $\mathrm{x}$ & $\mathrm{x}$ & $\mathrm{x}$ \\
\hline
\end{tabular}

${ }^{*}$ Cancer recurrence will be diagnosed at an unscheduled hospital visit when clinical symptomatology appears. 
is recommended that head CT/MRI, bone scintigraphy and positron emission tomography examination be performed at 1 year and 2 years after surgery. In addition, if clinical symptoms or other laboratory tests suggest postoperative recurrence, it is recommended that active testing be performed as needed.

\section{Primary, secondary and safety endpoints}

The primary endpoint is recurrence-free survival (RFS), which is defined as the time from randomisation until recurrence or death due to any cause. Patients without an event were censored at the time of the final follow-up. The secondary endpoint is OS, which is defined as the time from randomisation until death due to any cause. Although OS (or the 5-year survival rate) has been used to evaluate the treatment effects of resectable NSCLC, it has recently been reported that RFS can be a surrogate endpoint for OS. ${ }^{37}$ It was shown that recurrence in lung cancer peaked within 1 year after surgery and that over $60 \%$ of all recurrences occurred within 2 years after surgery. ${ }^{6}$ In a breast cancer study, ${ }^{38}$ a suppressive effect of perioperative NSAIDs on recurrence was shown at up to 2 years after surgery; that is, it may not contribute to the suppression of recurrence after 2 years. Therefore, RFS was set as the primary endpoint.

The safety endpoints are perioperative complications occurring during surgery and within 30 days after surgery; (1) cardiovascular complications, (2) respiratory complications and (3) other complications. Adverse events are evaluated using the Common Terminology Criteria for Adverse Events V.5.0. ${ }^{39}$

\section{Data collection, monitoring and the data and safety monitoring committee}

For patient registration, randomisation and data collection, we used a web-based system established by the Internet Data and Information Center for Medical Research at the University Hospital Medical Information Network. The Data and Safety Monitoring Committee reviews the safety data and monitors protocol compliance and on-schedule study progress. Early termination of the trial can be considered if treatment-related death or serious adverse events occur.

\section{Sample size}

In a retrospective cohort study, ${ }^{6}$ including the institutions participating in this study, the 2-year RFS rate in patients with NSCLC with surgery was 0.75 . The HR of ketorolac administration in patients with surgically treated breast and lung cancer was $0.16-0.37 .{ }^{18}{ }^{31}$ In addition, the HR of flurbiprofen administration in patients with surgically treated lung cancer was $0.80 .{ }^{28}$ In this study, we assumed that the 2-year RFS rate in the no-NSAIDS group would be 0.75 and that the HR would be 0.60 . When the registration period is 2 years, the follow-up period is 2 years, the one-sided $\alpha$ is set to $5 \%$ and the power is set to $80 \%$, the required number of patients is 382 . Considering deviations and loss to follow-up, the target number of patients was set to 420 (210 per group).

\section{Statistical analyses}

The primary analysis was to evaluate the treatment effect of flurbiprofen axetil on RFS. Survival curves for RFS will be compared between two groups by the stratified logrank test with allocation factors (sex, clinical stage) other than institutions. The Kaplan-Meier method is used to estimate survival curves for RFS, median RFS and 2-year RFS. To estimate the HR as a treatment effect, a stratified Cox proportional hazard model will be established using the same factors as the log-rank test. The one-sided significance level is set to $5 \%$ for the log-rank test, and the twosided confidence level for interval estimation is set to $5 \%$.

The Full Analysis Set (FAS) will be defined as all patients randomly assigned to a treatment group with at least one efficacy assessment after randomisation, and the Per Protocol Set (PPS) will be defined as all patients in the FAS who do not have major protocol deviations. The Safety Analysis Set (SAS) will include all randomised patients with at least one safety assessment after randomisation. The FAS will be used for all efficacy analyses, and the PPS will be used for sensitivity analysis, while the SAS will be used for the safety analysis. Missing data will not be imputed and instead be excluded.

In the subgroup analysis, we will consider the following factors: sex, age, smoking, body mass index, surgical approach, anaesthetic technique, analgesic agents, infectious complications, $\mathrm{C}$ reactive protein (CRP), NLR, Glasgow prognostic score (GPS), modified GPS, platelet to lymphocyte ratio, CRP to albumin ratio, advanced lung cancer inflammation index.

\section{Patient and public involvement}

The patients and public were not involved in the design, patient recruitment or conduct of the study. However, the study findings will be disseminated to the public and patients through multiple channels including publication, meetings, conferences and social media.

\section{Ethics and dissemination}

The study protocol V.1.0 was approved by Clinical Research Review Board of Saitama Medical University in September 2019 (No. 192002) and will be approved by each institutional review board of all participating institutions before patient enrolment. This study complies with the latest version of the Declaration of Helsinki, Clinical Trial Act and related notifications. Written informed consent will be obtained from all patients before registration. Personal information will remain confidential, and data will be de-identified using participant numbers.

\section{DISCUSSION}

To date, no complete randomised controlled trials have assessed the potential effects of NSAIDs on postoperative recurrence in NSCLC. Surgery is the main treatment for 
early stage NSCLC. However, surgical procedures accompanied by tissue injury can induce acute inflammation by causing the production of cytokines, prostaglandins and VEGF, which promote neoplastic progression. Thus, surgery itself may play a fundamental role in cancer recurrence. It has been reported that anaesthetic technique and analgesic agents administered during surgery might influence cancer recurrence. Flurbiprofen axetil is a safe and inexpensive NSAID that is used perioperatively as a part of multimodal analgesia. Unlike most cytotoxic chemotherapies, this treatment can be safely used perioperatively to reduce postoperative recurrence. Results from this study will provide clinically valuable information.

\section{Ethics approval}

This study was approved by the Clinical Research Review Boards of Saitama Medical University (approved number 192002) for all institutes.

Acknowledgements Yokohama Consortium of Thoracic Surgeons members and The International Lung-Clinical-Study Organisation members: Dr Yoshihiro Ishikawa, Yokohama City University Hospital; Dr Michihiko Tajiri, Kanagawa Cardiovascular and Respiratory Center; Dr Hiromasa Arai, Kanagawa Cardiovascular and Respiratory Center; Dr Takao Morohoshi, Yokosuka Kyosai Hospital; Dr Kohei Ando, Yokosuka Kyosai Hospital; Dr Hiroyuki Osawa, Yokohama Minami Kyosai Hospital; Dr Kazuki Yamanaka, Hiratsuka Kyosai Hospital; Dr Taketsugu Yamamoto, Yokohama Rosai Hospital; Dr Atsuo Gorai, Kanto Rosai Hospital; Dr Takahiro Omori, Yokosuka General Hospital Uwamachi; Dr Daisuke Noma, Saiseikai Yokohamashi Nanbu Hospital; Dr Kazuhito Funai, Hamamatsu University Hospital; Dr Akikazu Kawase, Hamamatsu University Hospital; Dr Noriyuki Matsutani, Teikyo University Mizonokuchi Hospital; Dr Nobumasa Takahashi, Saitama Cardiovascular and Respiratory Center.

Contributors KS - study design, sample size and statistical analysis plan, drafting of the protocol and manuscript. KW—study design, preparation of study protocol and review of the manuscript, chief investigator for the trial. TW—study design, preparation of study protocol, review of the manuscript. MM—review of the manuscript.

Funding The authors have not declared a specific grant for this research from any funding agency in the public, commercial or not-for-profit sectors.

Competing interests None declared.

Patient and public involvement Patients and/or the public were not involved in the design or conduct plans of this research.

Patient consent for publication Not required.

Provenance and peer review Not commissioned; externally peer reviewed.

Open access This is an open access article distributed in accordance with the Creative Commons Attribution Non Commercial (CC BY-NC 4.0) license, which permits others to distribute, remix, adapt, build upon this work non-commercially, and license their derivative works on different terms, provided the original work is properly cited, appropriate credit is given, any changes made indicated, and the use is non-commercial. See: http://creativecommons.org/licenses/by-nc/4.0/.

\section{ORCID iD}

Katsuya Watanabe http://orcid.org/0000-0001-8898-0370

\section{REFERENCES}

1 Siegel RL, Miller KD, Jemal A. Cancer statistics, 2019. CA Cancer J Clin 2019;69:7-34.

2 Sawabata N, Miyaoka E, Asamura $\mathrm{H}$, et al. Japanese lung cancer registry study of 11,663 surgical cases in 2004: demographic and prognosis changes over decade. J Thorac Oncol 2011;6:1229-35.

3 Baggstrom MQ, Stinchcombe TE, Fried DB, et al. Third-generation chemotherapy agents in the treatment of advanced non-small cell lung cancer: a meta-analysis. J Thorac Oncol 2007;2:845-53.
4 Asamura H, Goya T, Koshiishi Y, et al. A Japanese lung cancer registry study: prognosis of 13,010 resected lung cancers. $J$ Thorac Oncol 2008;3:46-52.

5 Goya T, Asamura H, Yoshimura $\mathrm{H}$, et al. Prognosis of 6644 resected non-small cell lung cancers in Japan: a Japanese lung cancer registry study. Lung Cancer 2005;50:227-34.

6 Watanabe K, Tsuboi M, Sakamaki K, et al. Postoperative followup strategy based on recurrence dynamics for non-small-cell lung cancer. Eur J Cardiothorac Surg 2016;49:1624-31.

7 Sugimura $\mathrm{H}$, Nichols FC, Yang P, et al. Survival after recurrent nonsmall-cell lung cancer after complete pulmonary resection. Ann Thorac Surg 2007;83:409-18.

8 Wada H, Hitomi S, Teramatsu T. Adjuvant chemotherapy after complete resection in non-small-cell lung cancer. West Japan Study Group for lung cancer surgery. J Clin Oncol 1996;14:1048-54.

9 Hamada C, Tsuboi M, Ohta M, et al. Effect of postoperative adjuvant chemotherapy with tegafur-uracil on survival in patients with stage la non-small cell lung cancer: an exploratory analysis from a meta-analysis of six randomized controlled trials. J Thorac Oncol 2009;4:1511-6.

10 Arriagada R, Bergman B, Dunant A, et al. Cisplatin-based adjuvant chemotherapy in patients with completely resected non-small-cell lung cancer. N Engl J Med 2004;350:351-60.

11 Winton T, Livingston R, Johnson D, et al. Vinorelbine plus cisplatin vs. observation in resected non-small-cell lung cancer. $N$ Engl $J$ Med 2005;352:2589-97.

12 Douillard J-Y, Rosell R, De Lena M, et al. Adjuvant vinorelbine plus cisplatin versus observation in patients with completely resected stage IB-IIIA non-small-cell lung cancer (Adjuvant Navelbine International Trialist Association [ANITA]): a randomised controlled trial. Lancet Oncol 2006;7:719-27.

13 Balkwill F, Mantovani A. Inflammation and cancer: back to Virchow? Lancet 2001;357:539-45.

14 Coussens LM, Werb Z. Inflammation and cancer. Nature 2002;420:860-7.

15 Oshima H, Nakayama M, Han T-S, et al. Suppressing TGF $\beta$ signaling in regenerating epithelia in an inflammatory microenvironment is sufficient to cause invasive intestinal cancer. Cancer Res 2015;75:766-76

16 Hanahan D, Weinberg RA. Hallmarks of cancer: the next generation. Cell 2011:144:646-74.

17 Nojiri T, Hosoda H, Tokudome T, et al. Atrial natriuretic peptide prevents cancer metastasis through vascular endothelial cells. Proc Natl Acad Sci U S A 2015;112:4086-91.

18 Forget P, Machiels J-P, Coulie PG, et al. Neutrophil:lymphocyte ratio and intraoperative use of ketorolac or diclofenac are prognostic factors in different cohorts of patients undergoing breast, lung, and kidney cancer surgery. Ann Surg Oncol 2013;20:650-60.

19 Krall JA, Reinhardt F, Mercury OA, et al. The systemic response to surgery triggers the outgrowth of distant immune-controlled tumors in mouse models of dormancy. Sci Transl Med 2018;10:eaan3464.

20 Wong RSY. Role of nonsteroidal anti-inflammatory drugs (NSAIDs) in cancer prevention and cancer promotion. Adv Pharmacol Sci 2019;2019:1-10.

21 McCormack VA, Hung RJ, Brenner DR, et al. Aspirin and NSAID use and lung cancer risk: a pooled analysis in the International lung cancer Consortium (ILCCO). Cancer Causes Control 2011;22:1709-20.

22 Bowers LW, Maximo IXF, Brenner AJ, et al. NSAID use reduces breast cancer recurrence in overweight and obese women: role of prostaglandin-aromatase interactions. Cancer Res 2014;74:4446-57.

23 Wang X, Peters U, Potter JD, et al. Association of nonsteroidal anti-inflammatory drugs with colorectal cancer by subgroups in the vitamins and lifestyle (VITAL) study. Cancer Epidemiol Biomarkers Prev 2015;24:727-35.

24 Harris RE. Cyclooxygenase-2 (COX-2) blockade in the chemoprevention of cancers of the colon, breast, prostate, and lung. Inflammopharmacology 2009;17:55-67.

25 Wang K, Luo J, Zheng L, et al. Preoperative flurbiprofen axetil administration for acute postoperative pain: a meta-analysis of randomized controlled trials. J Anesth 2017;31:852-60.

26 Esme $\mathrm{H}$, Kesli R, Apiliogullari B, et al. Effects of flurbiprofen on CRP, TNF- $\alpha$, IL-6, and postoperative pain of thoracotomy. Int J Med Sci 2011;8:216-21.

27 Wen Y, Wang M, Yang J, et al. A comparison of fentanyl and flurbiprofen axetil on serum VEGF-C, TNF- $\alpha$, and IL-1B concentrations in women undergoing surgery for breast cancer. Pain Pract 2015;15:530-7.

28 Huang W-W, Zhu W-Z, Mu D-L, et al. Perioperative management may improve long-term survival in patients after lung cancer surgery: a retrospective cohort study. Anesth Analg 2018;126:1666-74. 
29 Monk TG, Saini V, Weldon BC, et al. Anesthetic management and one-year mortality after noncardiac surgery. Aneth Analg 2005;100:4-10.

30 Hiller JG, Perry NJ, Poulogiannis G, et al. Perioperative events influence cancer recurrence risk after surgery. Nat Rev Clin Oncol 2018;15:205-18.

31 Forget $\mathrm{P}$, Vandenhende J, Berliere $\mathrm{M}$, et al. Do intraoperative analgesics influence breast cancer recurrence after mastectomy? A retrospective analysis. Anesth Analg 2010;110:1630-5.

32 Forget $\mathrm{P}$, Berlière $\mathrm{M}$, Maanen $\mathrm{AV}$, et al. Ketorolac in breast cancer trial (KBCtrial) group (2013) perioperative ketorolac in high risk breast cancer patients. rationale, feasibility and methodology of a prospective randomized placebo-controlled trial. Med Hypotheses 2013;81:707-12.

33 Brierly JD, Gospodarowicz MK, Wittekind C. TNM classification of malignant tumours. 8th edn. Chichester, West Sussex, UK: WileyBlackwell, 2017.

34 Visbal AL, Williams BA, Nichols FC, et al. Gender differences in non-small-cell lung cancer survival: an analysis of 4,618 patients diagnosed between 1997 and 2002. Ann Thorac Surg 2004;78:209-15.

35 Sakurai H, Asamura H, Goya T, et al. Survival differences by gender for resected non-small cell lung cancer: a retrospective analysis of 12,509 cases in a Japanese lung cancer registry study. J Thorac Oncol 2010;5:1594-601.

36 The Japan Lung Cancer Society. Guidelines for diagnosis and treatment of the lung cancer 2018. Tokyo: Kanehara \& co., Ltd, 2018.

37 Mauguen A, Pignon J-P, Burdett S, et al. Surrogate endpoints for overall survival in chemotherapy and radiotherapy trials in operable and locally advanced lung cancer: a re-analysis of meta-analyses of individual patients' data. Lancet Oncol 2013;14:619-26.

38 Retsky M, Demicheli R, Hrushesky WJM, et al. Reduction of breast cancer relapses with perioperative non-steroidal anti-inflammatory drugs: new findings and a review. Curr Med Chem 2013;20:4163-76.

39 Japan Clinical Oncology Group (JCOG). Common terminology criteria for adverse events version 5.0, 2017. Available: http://www. jcog.jp/doctor/tool/CTCAEv5J_20190905_v22_1.pdf [Accessed 1 Apr 2020]. 